\title{
IDENTITIES RELATED TO THE STIRLING NUMBERS AND MODIFIED APOSTOL-TYPE NUMBERS ON UMBRAL CALCULUS
}

\author{
TAKAO KOMATSU AND YILMAZ SIMSEK \\ Received 07 December, 2014
}

\begin{abstract}
In this paper, by using umbral calculus and umbral algebra methods, we derive several interesting identities and relations related to the modified and unification of the Bernoulli, Euler and Genocchi polynomials and numbers and the generalized ( $\beta$-) Stirling numbers of the second kind. We also give some applications and remarks related to these numbers and polynomials.
\end{abstract}

2010 Mathematics Subject Classification: 11B68; 11S40; 05A19; 11S80; 11M99; 30B50; 44A05

Keywords: Bernoulli numbers and polynomials, Euler numbers and polynomials, Genocchi Bernoulli numbers and polynomials, Stirling numbers, Umbral calculus, Umbral algebra

\section{INTRODUCTION, DEFINITIONS AND PRELIMINARIES}

As usual, let $\mathbb{N}, \mathbb{Z}, \mathbb{Q}, \mathbb{R}, \mathbb{R}^{+}$and $\mathbb{C}$ denote the sets of positive integers, integers, rational numbers, real numbers, positive real numbers and complex numbers, respectively, and $\mathbb{N}_{0}:=\mathbb{N} \cup\{0\}$. Assume that $\log z$ denotes the principal branch of the multi-valued function $\log z$ with the imaginary part $\Im(\log z)$ constrained by $-\pi<\Im(\log z)<\pi$. Denote the falling factorial by $(n)_{k}=n(n-1) \cdots(n-k+1)$ $(k \geq 1)$ with $(n)_{0}=1$.

The unification of the Bernoulli, Euler and Genocchi polynomials is defined by Ozden [17]:

$$
g_{\beta}(x, t ; k, a, l):=\frac{2^{1-k} t^{k} e^{t x}}{\beta^{l} e^{t}-a^{l}}=\sum_{n=0}^{\infty} y_{n, \beta}(x ; k, a, l) \frac{t^{n}}{n !} .
$$

If $\beta=a$ then $|t|<2 \pi$, and if $\beta \neq a, k \in \mathbb{N}_{0}$ and $a, l \in \mathbb{C} \backslash\{0\}$ then $|t|<l \log \left(\frac{\beta}{a}\right)$.

Remark 1. Note that Equation (1.1) with $x=1$ is reduced to the generating functions for the unification of the Bernoulli, Euler and Genocchi numbers.

\footnotetext{
The first author was supported in part by the grant of Wuhan University and Hubei Provincial Pro-
} gram.

The second author was supported by Akdeniz University Scientific Research Projects Unit. 
Remark 2. Using the special values of $a, l, k$ and $\beta$ in (1.1), the polynomials $y_{n, \beta}(x ; k, a, l)$ provide us a generalization and unification of the Apostol-Bernoulli polynomials, Apostol-Euler polynomials and Apostol-Genocchi polynomials, given by

$$
\begin{aligned}
& \mathcal{B}_{n}(x, \beta)=y_{n, \beta}(x ; 1,1,1), \\
& \mathcal{E}_{n}(x, \beta)=y_{n, \beta}(x ; 0,-1,1)
\end{aligned}
$$

and

$$
\mathcal{E}_{n}(x, \beta)=y_{n, \beta}(x ; 1,-1,1),
$$

respectively. Moreover, for the classical Bernoulli polynomials $B_{n}(x)$, the classical Euler polynomials $E_{n}(x)$ and the classical Genocchi polynomials $G_{n}(x)$, given by

$$
\begin{gathered}
B_{n}(x)=\mathscr{B}_{n}(x, 1), \\
E_{n}(x)=\mathcal{E}_{n}(x, 1)
\end{gathered}
$$

and

$$
G_{n}(x)=\mathscr{E}_{n}(x, 1),
$$

respectively. Substituting $x=0$ into these polynomials, one also has the classical Bernoulli numbers $B_{n}$, the classical Euler numbers $E_{n}$ and the classical Genocchi numbers $G_{n}$, given by

$$
\begin{aligned}
& B_{n}=B_{n}(0), \\
& E_{n}=E_{n}(0),
\end{aligned}
$$

and

$$
G_{n}=G_{n}(0),
$$

respectively. In [9], Bernoulli polynomial can be expressed as an affine combination of the polynomials $(x-1)^{n},(x-2)^{n}, \ldots,(x-k-1)^{n}$. In [25], generating functions for some families of Genocchi type polynomials were constructed. In [8], a further investigation for the Apostol-Bernoulli polynomials and the Apostol-Genocchi polynomials was presented. In [7], some new formulas for such a kind of sums of the products of an arbitrary number of the Apostol-Bernoulli, Euler, and Genocchi polynomials were established by making use of the generating function methods and summation transform techniques. See [1]-[26] and the references therein.

In [15], Ozden and Simsek modified the polynomials $y_{n, \beta}(x ; k, a, l)$ as follows:

$$
f(t ; k, a, b, \beta)=\left(\frac{t^{k} 2^{1-k}}{\beta b^{t}-a^{t}}\right)^{v} b^{x t}=\sum_{n=0}^{\infty} Y_{n, \beta}^{(v)}(x, k, a, b) \frac{t^{n}}{n !},
$$

where the polynomials $Y_{n, \beta}^{(v)}(x, k, a, b)$ are called modification and unification of the Apostol-type polynomials of order $v$. One easily sees that

$$
Y_{n, \beta}^{(v)}(0, k, a, b)=Y_{n, \beta}^{(v)}(k, a, b),
$$


which denotes modification and unification of the Apostol-type numbers of order $v$, and

$$
y_{n, \beta}^{(v)}(x ; k, 1,1)=Y_{n, \beta}^{(v)}(x, k, 1, e)
$$

which denotes Apostol-type polynomials (See [16, 17]). In [11], several symmetry identities for generalized Apostol type polynomials were proved by using their generating functions.

Ozden and Simsek [15] gave an explicit formula for the polynomials $Y_{n, \beta}^{(v)}(x, k, a, b)$ as

$$
Y_{n, \beta}^{(v)}(x, k, a, b)=\sum_{j=0}^{n}\left(\begin{array}{l}
n \\
j
\end{array}\right) x^{n-j}(x \ln b)^{n-j} Y_{j, \beta}^{(v)}(k, a, b) .
$$

and also gave the recurrence relation for the numbers $Y_{\beta}(k, a, b)$ as

$$
\beta\left(Y_{\beta}(k, a, b)+\ln b\right)^{n}-\left(Y_{\beta}(k, a, b)+\ln a\right)^{n}=\left\{\begin{array}{cc}
2^{1-k} k ! & n=k, \\
0 & n \neq k,
\end{array}\right.
$$

where $\left(Y_{\beta}(k, a, b)\right)^{m}$ is replaced by $Y_{m, \beta}(k, a, b)$.

Remark 3. $Y_{m, \beta}(k, a, b)$ is a generalization of the classical Bernoulli, Euler and Genocchi numbers. If we substitute $k=a=\beta=v=1$ and $b=e$ into (1.2), we have

$$
Y_{n, 1}^{(1)}(1,1, e)=B_{n}
$$

If we substitute $k=0, a=v=1, \beta=-1$ and $b=e$ into (1.2), we have

$$
Y_{n,-1}^{(1)}(0,1, e)=-E_{n} \text {. }
$$

If we substitute $k=a=v=1, \beta=-1$ and $b=e$ into (1.2), we have

$$
Y_{n,-1}^{(v)}(1,1, e)=-\frac{1}{2} G_{n} .
$$

The generalized $\beta$-Stirling type numbers of the second kind are given by the following definition:

Definition 1 (see [21]). Let $a, b \in \mathbb{R}^{+}$with $a \neq b, \beta \in \mathbb{C}$ and $v \in \mathbb{N}_{0}$. The generalized Stirling numbers of the second kind $\delta(n, v ; a, b ; \beta)$ are defined by the generating function

$$
f_{S, v}(t ; a, b ; \beta)=\frac{\left(\beta b^{t}-a^{t}\right)^{v}}{v !}=\sum_{n=0}^{\infty} \delta(n, v ; a, b ; \beta) \frac{t^{n}}{n !} .
$$

Substituting $a=1$ and $b=e$ into (1.3), we have the $\beta$-Stirling numbers of the second kind

$$
\S(n, v ; 1, e ; \beta)=S(n, v ; \beta)
$$


(See $[13,23,24]$ ). If $\beta=1$, then we get the classical Stirling numbers of the second kind by

(See [1]-[26]).

$$
S(n, v ; 1)=S(n, v)
$$

We have the following theorem in [22].

Theorem 1. We have

$$
\S(n, v ; a, b ; \beta)=\frac{1}{v !} \sum_{j=0}^{v}(-1)^{j}\left(\begin{array}{c}
v \\
j
\end{array}\right) \beta^{v-j}(j \ln a+(v-j) \ln b)^{n}
$$

and

$$
\S(n, v ; a, b ; \beta)=\frac{1}{v !} \sum_{j=0}^{v}(-1)^{v-j}\left(\begin{array}{c}
v \\
j
\end{array}\right) \beta^{j}(j \ln b+(v-j) \ln a)^{n} .
$$

Remark 4. Note that by setting $a=1$ and $b=e$ in the identity (1.4), we have

$$
S(n, v ; \beta)=\frac{1}{v !} \sum_{j=0}^{v}\left(\begin{array}{c}
v \\
j
\end{array}\right) \beta^{v-j}(-1)^{j}(v-j)^{n} .
$$

This relation has been studied by Srivastava [23] and Luo [13]. If $\beta=1$, then this relation is further reduced to

$$
S(n, v)=\frac{1}{v !} \sum_{j=0}^{v}\left(\begin{array}{c}
v \\
j
\end{array}\right)(-1)^{j}(v-j)^{n}
$$

(see [1]-[26]).

Definition 2 ([22]). Let $a, b \in \mathbb{R}^{+}$with $a \neq b, x \in \mathbb{R}, \beta \in \mathbb{C}$ and $v \in \mathbb{N}_{0}$. The generalized array type polynomials $\delta_{v}^{n}(x ; a, b ; \beta)$ are defined by means of the following generating function:

$$
g_{v}(x, t ; a, b ; \beta)=\frac{1}{v !}\left(\beta b^{t}-a^{t}\right)^{v} b^{x t}=\sum_{n=0}^{\infty} 8_{v}^{n}(x ; a, b ; \beta) \frac{t^{n}}{n !} .
$$

By using (1.6), we have

$$
\wp_{v}^{n}(x ; a, b ; \beta)=\frac{1}{v !} \sum_{j=0}^{v}(-1)^{v-j}\left(\begin{array}{c}
v \\
j
\end{array}\right) \beta^{j}\left(\ln \left(a^{v-j} b^{x+j}\right)\right)^{n}
$$

(see [22]).

Note that the polynomials $\wp_{v}^{n}(x ; a, b ; \beta)$ are called the generalized $\lambda$-array type polynomials.

Substituting $x=0$ into (1.7), we get (1.5) by

$$
\delta_{v}^{n}(0 ; a, b ; \beta)=8(n, v ; a, b ; \beta) .
$$


Setting $a=\beta=1$ and $b=e$ in (1.7), we have

$$
S_{v}^{n}(x)=\frac{1}{v !} \sum_{j=0}^{v}(-1)^{v-j}\left(\begin{array}{l}
v \\
j
\end{array}\right)(x+j)^{n},
$$

which is a result in Chang and Ha [2, Eq.(3.1)] and Simsek [21]. It is easy to see that $S_{0}^{0}(x)=S_{n}^{n}(x)=1, S_{0}^{n}(x)=x^{n}$, and for $v>n, S_{v}^{n}(x)=0$ (see [2, Eq.(3.1)]).

Theorem 2 ([22]). We have

$$
\delta_{v}^{n}(x ; a, b ; \lambda)=\sum_{j=0}^{n}\left(\begin{array}{c}
n \\
j
\end{array}\right) \&(j, v ; a, b ; \lambda)\left(\ln b^{x}\right)^{n-j} .
$$

The theory of the umbral calculus and the umbral algebra has been applied and expanded by many authors. By using the action of a linear functional on a polynomial Sheffer sequences and Appell sequences, one can obtain many properties of various polynomials. In [4] by using methods of umbral calculus and algebraic nature, some progress in the theory of generating functions involving harmonic numbers were made. In [18] by investigating some umbral-calculus generalizations of the addition formulas, several addition formulas for a general class of Appell sequences are given, involving the generalized Bernoulli, Euler, Genocchi and Srivastava polynomials. In [26] umbral calculus presentations of the Chan-Chyan-Srivastava polynomials and of their substantially more general form, the Erkuş-Srivastava polynomials were investigated.

In this paper, by using umbral calculus and umbral algebra methods, we derive several interesting identities and relations related to the modified and unification of the Bernoulli, Euler and Genocchi polynomials and numbers and the generalized ( $\beta$ ) Stirling numbers of the second kind. We also give some applications and remarks related to these numbers and polynomials.

Some results in this paper are extracted from the preprint [20], and the conference proceeding and survey can be seen in [10].

\section{IDENTITIES ON UMBRAL CALCULUS AND UMBRAL ALGEBRA}

In this section, we give several relations between the modification and unification of the Apostol-type polynomials of order $v$ and the Stirling numbers of the second kind.

We need some identities of the umbral algebra and calculus. The following formulas and notations are given in work of Roman [19].

Let $P$ be the algebra of polynomials in the single variable $x$ over the complex number field. Let $P^{*}$ be the vector space of all linear functionals on $P$. Let $\langle L \mid p(x)\rangle$ be the action of a linear functional $L$ on a polynomial $p(x)$. Let $F$ denote the algebra 
of formal power series

$$
f(t)=\sum_{k=0}^{\infty} \frac{a_{k}}{k !} t^{k} .
$$

Let $f \in F$ define a linear functional on $P$ and for all $k \in \mathbb{N}_{0}$,

$$
a_{k}=\left\langle f(t) \mid x^{k}\right\rangle .
$$

The order $o(f(t))$ of a power series $f(t)$ is the smallest integer $k$ for which the coefficient of $t^{k}$ does not vanish. A series $f(t)$ for which $o(f(t))=1$ is called a delta series. And a series $f(t)$ for which $o(f(t))=0$ is called an invertible series.

Let $f(t), g(t)$ be in $F$. Then we have

$$
\langle f(t) g(t) \mid p(x)\rangle=\langle f(t) \mid g(t) p(x)\rangle .
$$

For all $p(x)$ in $P$, we have

$$
\left\langle e^{y t} \mid p(x)\right\rangle=p(y)
$$

and

$$
e^{y t} p(x)=p(x+y) .
$$

The Sheffer polynomials are defined by the generating function

$$
\sum_{k=0}^{\infty} \frac{s_{k}(x)}{k !} t^{k}=\frac{1}{g(t)} e^{x t} .
$$

(see [19, Theorem 2.3.4]).

Theorem 3 ([19, Theorem 2.3.6]). Let $f(t)$ be a delta series and let $g(t)$ be an invertible series. Then there exist a unique sequence $s_{n}(x)$ of polynomials satisfying the orthogonality condition

$$
\left\langle g(t) f(t)^{k} \mid s_{n}(x)\right\rangle=n ! \delta_{n, k}
$$

for all $n, k \in \mathbb{N}_{0}$.

Let

$$
s_{n}(x)=g(t)^{-1} x^{n}
$$

with the derivative formula

$$
t s_{n}(x)=n s_{n-1}(x) .
$$

We can obtain the following lemma.

Lemma 1. For $n \in \mathbb{N}_{0}$, we have

$$
Y_{n, \beta}^{(v)}(x ; k, a, b)=\left(\frac{2^{1-k} t^{k}}{\beta b^{t}-a^{t}}\right)^{v}(\ln b)^{n} x^{n} .
$$


Proof. By (1.2)

$$
\begin{aligned}
\sum_{n=0}^{\infty} Y_{n, \beta}^{(v)}(x, k, a, b) \frac{t^{n}}{n !} & =\left(\frac{t^{k} 2^{1-k}}{\beta b^{t}-a^{t}}\right)^{v} e^{x t \ln b} \\
& =\left(\frac{t^{k} 2^{1-k}}{\beta b^{t}-a^{t}}\right)^{v} \sum_{n=0}^{\infty}(x \ln b)^{n} \frac{t^{n}}{n !}
\end{aligned}
$$

Applying (2.2) with (2.1) to both sides of this equation, we get the result.

Lemma 2. For $n \in \mathbb{N}_{0}$, we have

$$
\begin{aligned}
\left\langle\left(\beta b^{t}-a^{t}\right)^{j} \mid Y_{n, \beta}(x ; k, a, b)\right\rangle & \\
= & \sum_{m=0}^{j}\left(\begin{array}{c}
j \\
m
\end{array}\right)(-1)^{j-m} \beta^{m} Y_{n, \beta}(m \ln b+(j-m) \ln a, k, a, b) .
\end{aligned}
$$

Proof.

$$
\begin{aligned}
& \left\langle\left(\beta b^{t}-a^{t}\right)^{j} \mid Y_{n, \beta}(x ; k, a, b)\right\rangle \\
& =\left\langle\sum_{m=0}^{j}(-1)^{j-m}\left(\begin{array}{c}
j \\
m
\end{array}\right) \beta^{m} e^{t(m \ln b+(j-m) \ln a)} \mid Y_{n, \beta}(x ; k, a, b)\right\rangle \\
& =\sum_{m=0}^{j}(-1)^{j-m}\left(\begin{array}{c}
j \\
m
\end{array}\right) \beta^{m}\left\langle e^{t(m \ln b+(j-m) \ln a)} \mid Y_{n, \beta}(x ; k, a, b)\right\rangle .
\end{aligned}
$$

Substituting Equation (2.4) into the above equation, we get the desired result.

Remark 5. We see that

$$
\left\langle\left(\beta b^{t}-1\right)^{j} \mid Y_{n, \beta}(x ; k, 1, b)\right\rangle=\left\langle\left(\beta e^{t}-1\right)^{j} \mid y_{n, \beta}(x, k, 1,1)\right\rangle
$$

([6, Lemma 2. Eq.(3.1)]).

Lemma 3. We have

$$
v^{n} S(n, l)=\frac{1}{l !}\left\langle\left(e^{v t}-1\right)^{l} \mid x^{n}\right\rangle,
$$

where $S(n, l)$ are the Stirling numbers of the second kind.

Proof. Since

$$
\frac{1}{l !}\left(e^{v t}-1\right)^{l}=\sum_{j=0}^{\infty} v^{n} S(n, l) \frac{t^{n}}{n !},
$$

by using (2.1) and (2.2), we get the desired result. 
Remark 6. Substituting $v=1$ into Lemma 3, we have

$$
S(n, l)=\frac{1}{l !}\left\langle\left(e^{t}-1\right)^{l} \mid x^{n}\right\rangle
$$

$([19, \mathrm{p} .59])$

Theorem 4. We have

$$
\begin{aligned}
& \sum_{m=0}^{j}\left(\begin{array}{c}
j \\
m
\end{array}\right)(-1)^{j-m} \beta^{m} Y_{n, \beta}(m \ln b+(j-m) \ln a, k, a, b) \\
& =2^{1-k}(\ln b)^{n} \beta^{j-1} k !\left(\begin{array}{l}
n \\
k
\end{array}\right) \sum_{l=0}^{j-1} l !\left(\begin{array}{c}
j-1 \\
l
\end{array}\right)\left(1-\frac{1}{\beta}\right)^{j-l-1} \\
& \quad \times \sum_{v=0}^{n-k}\left(\begin{array}{c}
n-k \\
v
\end{array}\right)((j-1) \ln a)^{n-k-v}\left(\ln \frac{b}{a}\right)^{v} S(v, l) .
\end{aligned}
$$

Proof. By Lemma 1, we get

$$
\left\langle\left(\beta b^{t}-a^{t}\right)^{j} \mid Y_{n, \beta}(x ; k, a, b)\right\rangle=\left\langle\left(\beta b^{t}-a^{t}\right)^{j} \mid \frac{2^{1-k} t^{k}}{\beta b^{t}-a^{t}}(\ln b)^{n} x^{n}\right\rangle .
$$

Substituting (2.3) and (2.9) into this equation and applying Lemma 2, we get

$$
\left\langle\left(\beta b^{t}-a^{t}\right)^{j} \mid Y_{n, \beta}(x ; k, a, b)\right\rangle=2^{1-k}(\ln b)^{n}\left\langle\left(\beta b^{t}-a^{t}\right)^{j-1} \mid k !\left(\begin{array}{l}
n \\
k
\end{array}\right) x^{n-k}\right\rangle .
$$

After some elementary calculations in this equation, we obtain

$$
\begin{aligned}
& \left\langle\left(\beta b^{t}-a^{t}\right)^{j} \mid Y_{n, \beta}(x ; k, a, b)\right\rangle \\
& =2^{1-k}(\ln b)^{n} k !\left(\begin{array}{l}
n \\
k
\end{array}\right) \beta^{j-1} \sum_{l=0}^{j-1}\left(\begin{array}{c}
j-1 \\
l
\end{array}\right)\left(1-\frac{1}{\beta}\right)^{j-l-1} e^{t((j-1) \ln a)} \\
& \quad \times\left\langle\left(e^{t\left(\ln \frac{b}{a}\right)}-1\right)^{l} \mid x^{n-k}\right\rangle .
\end{aligned}
$$

By applying Lemma 3 with (2.3) in this equation, we obtain the desired result.

Remark 7. Substituting $a=1$ and $b=e$ into the identity in Theorem 4 , we get the work of Dere et al. [6, Theorem 3, Corollary 2]

$$
\sum_{m=0}^{j}\left(\begin{array}{c}
j \\
m
\end{array}\right)(-1)^{j-m} \beta^{m} \mathcal{Y}_{n, \beta}(m ; k, 1,1)
$$




$$
=\frac{\beta^{j-1}}{2^{k-1}} k !\left(\begin{array}{l}
n \\
k
\end{array}\right) \sum_{l=0}^{j-1} \frac{(j-1) !}{(j-l-1) !}\left(1-\frac{1}{\beta}\right)^{j-l-1} S(n-k, l) .
$$

Remark 8. By setting $\beta=k=a=1$ and $b=e$ in Theorem 4, we get the following well-known result in [19, P. 94]:

$$
\left\langle\left(e^{t}-1\right)^{j} \mid B_{n}(x)\right\rangle=n(j-1) ! S(n-1, j-1)
$$

or

$$
\sum_{m=0}^{j}\left(\begin{array}{c}
j \\
m
\end{array}\right)(-1)^{j-m} B_{n}(m)=n(j-1) ! S(n-1, j-1) .
$$

Substituting $a=1, k=0, \beta=-1$ and $b=e$ into the identity in Theorem 4, we get the following:

\section{Corollary 1.}

$$
\sum_{m=0}^{j}\left(\begin{array}{c}
j \\
m
\end{array}\right) E_{n}(m)=\sum_{l=0}^{j-1}\left(\begin{array}{c}
j-l \\
l
\end{array}\right) 2^{j-l} l ! S(n, l) .
$$

Substituting $a=1, k=1, \beta=-1$ and $b=e$ into Theorem 4, we get the following results:

\section{Corollary 2.}

$$
\sum_{m=0}^{j}\left(\begin{array}{c}
j \\
m
\end{array}\right) G_{n}(m)=n \sum_{l=0}^{j-1}\left(\begin{array}{c}
j-l \\
l
\end{array}\right) 2^{j-l-2} l ! S(n-1, l) .
$$

\section{Corollary 3.}

$$
\left\langle\left(e^{t}+1\right)^{j} \mid G_{n}(x)\right\rangle=\sum_{m=0}^{j}\left(\begin{array}{c}
j \\
m
\end{array}\right) G_{n}(m) .
$$

Proof. In the work of Dere et al. [6, p. 3255 ], it is proved that

$$
\begin{aligned}
\left\langle\left(e^{t}-1\right)^{j} \mid y_{n,-1}(x, 1,-1, e)\right\rangle & =\left\langle\left(e^{t}+1\right)^{j} \mid G_{n}(x)\right\rangle \\
& =n \sum_{l=0}^{j-1}\left(\begin{array}{c}
j-l \\
l
\end{array}\right) 2^{j-l} l ! S(n-1, l) .
\end{aligned}
$$

Combining this equation with Corollary 2 , we have the desired result.

Theorem 5. (Recurrence relation) For $v \geq 2$, we have

$$
\begin{aligned}
\beta Y_{n, \beta}^{(v)}(x+\ln b ; k, a, b)= & Y_{n, \beta}^{(v)}(x+\ln a ; k, a, b) \\
& +2^{1-k}(n)_{k}(\ln b)^{k} Y_{n-k, \beta}^{(v-1)}(x ; k, a, b) .
\end{aligned}
$$


Proof. By using Lemma 1, we get

$$
\left(\beta b^{t}-a^{t}\right) Y_{n, \beta}^{(v)}(x ; k, a, b)=2^{1-k}(n)_{k}(\ln b)^{k} Y_{n-k, \beta}^{(v-1)}(x ; k, a, b) .
$$

By using (2.5), we obtain

$$
\left(\beta b^{t}-a^{t}\right) Y_{n, \beta}^{(v)}(x ; k, a, b)=\beta Y_{n, \beta}^{(v)}(x+\ln b ; k, a, b)-Y_{n, \beta}^{(v)}(x+\ln a ; k, a, b) .
$$

By combining (2.10) and (2.11), we get the desired result.

Remark 9. If we set $a=1$ and $b=e$ in Theorem 5, we obtain

$$
\beta y_{n, \beta}^{(v)}(x+1 ; k, 1,1)=y_{n, \beta}^{(v)}(x ; k, 1,1)+2^{1-k}(n)_{k} y_{n-k, \beta}^{(v-1)}(x ; k, 1,1)
$$

([6, Theorem 6]).

Remark 10. By substituting $a=1, k=0, b=e$ and $\beta=-1$ into the identity in Theorem 5, we get the recurrence relations for the Euler polynomials of higher-order:

$$
E_{n}^{(v)}(x+1)=-E_{n}^{(v)}(x)+2 E_{n}^{(v-1)}(x) \quad(v \geq 2, n \in \mathbb{N})
$$

([6], [19, p.103]).

Remark 11. By substituting $a=k=1, b=e$ and $\beta=-1$ into the identity in Theorem 5, we have the recurrence relation for the Genocchi polynomials of higherorder:

$$
\left(e^{t}+1\right) G_{n}^{(v)}(x+1)=2 n G_{n-1}^{(v-1)}(x) \quad(v \geq 2, n \in \mathbb{N})
$$

([5, Theorem7])

Remark 12. By substituting $a=k=1, b=e$ and $\beta=1$ into the identity in Theorem 5, we have the recurrence relation for the Bernoulli polynomials of higherorder:

$$
B_{n}^{(v)}(x+1)=B_{n}^{(v)}(x)+n B_{n-1}^{(v-1)}(x) \quad(v \geq 2, n \in \mathbb{N})
$$

([6] and [19, Eq.(4.2.6)]).

By using Lemma 1 with (2.10), for $v=1$, we get the following theorem, which is very useful in the theory of the Diophantine equation:

Theorem 6. For $n, k \in \mathbb{N}_{0}$ with $n \geq k$, we have

$$
\left(\beta b^{t}-a^{t}\right) Y_{n, \beta}(x ; k, a, b)=2^{1-k}(n)_{k}(\ln b)^{k} x^{n-k} .
$$

Remark 13. Substituting $a=1$ and $b=e$ into (2.12), we have

$$
\beta y_{n, \beta}(x+1 ; k, 1, e)-y_{n, \beta}(x ; k, 1, e)=2^{1-k}(n)_{k} x^{n-k}
$$

([14]). By substituting $a=k=1, b=e$ and $\beta=1$ into (2.12), we have

$$
B_{n}(x+1)-B_{n}(x)=n x^{n-1}
$$

([14], [19, p.95], [24]). By substituting $a=1, k=0, b=e$ and $\beta=-1$ into (2.12), we have

$$
E_{n}(x+1)+E_{n}(x)=2 x^{n}
$$


([14], [19, p. 95], [24]). By substituting $a=k=1, b=e$ and $\beta=-1$ into (2.12), we have

$$
G_{n}(x+1)+G_{n}(x)=2 n x^{n}
$$

([5, Corollary 1], [14]).

\section{ACKNOWLEDGEMENT}

The authors thank the anonymous referee to show us a related article [12], which appeared independently during the long process of this article, as well as the additional references $[3,7,8,11,25,26]$.

\section{REFERENCES}

[1] T. M. Apostol, “On the Lerch zeta function.” Pacific J. Math., vol. 1, pp. 161-167, 1951, doi: $10.1137 / 050641867$.

[2] C.-H. Chang and C.-W. Ha, "A multiplication theorem for the Lerch zeta function and explicit representations of the Bernoulli and Euler polynomials." J. Math. Anal. Appl., vol. 315, no. 2, pp. 758-767, 2006, doi: 10.1137/050641867.

[3] G. Dattoli, B. Germano, S. Licciardi, and M. R. Martinelli, "On an umbral treatment of Gegenbauer, Legendre and Jacobi polynomials." preprint, vol. preprint, no. 2, pp. 423-441, 2007, doi: $10.1137 / 050641867$.

[4] G. Dattoli and H. M. Srivastava, "A note on harmonic numbers, umbral calculus and generating functions.” Appl. Math. Lett., vol. 21, no. 7, pp. 686-693, 2008, doi: 10.1137/050641867.

[5] R. Dere and Y. Simsek, "Genocchi polynomials associated with the Umbral algebra." Appl. Math. Comput., vol. 218, no. 3, pp. 756-761, 2011, doi: 10.1137/050641867.

[6] R. Dere, Y. Simsek, and H. M. Srivastava, "A unified presentation of three families of generalized Apostol type polynomials based upon the theory of the umbral calculus and the umbral algebra." J. Number Theory, vol. 133, no. 10, pp. 3245-3263, 2013, doi: 10.1137/050641867.

[7] Y. He, S. Araci, and H. M. Srivastava, "Some new formulas for the products of the Apostol type polynomials." Adv. Diff. Equ., vol. 2016, no. 287, p. 18, 2016, doi: 10.1186/s13662-016-1014-0.

[8] Y. He, S. Araci, H. M. Srivastava, and M. Acikgoz, "Some new identities for the Apostol-Bernoulli polynomials and the Apostol-Genocchi polynomials." Appl. Math. Comput., vol. 262, pp. 31-41, 2015, doi: 10.1016/j.amc.2015.03.132.

[9] T. Komatsu and C. de J. Pita Ruiz V., "Several explicit formulae for Bernoulli polynomials." Math. Commun., vol. 21, no. 1, pp. 127-140, 2016, doi: 10.1137/050641867.

[10] T. Komatsu and Y. Simsek, "Identities related to the Stirling numbers and modified Apostoltype numbers on Umbral Calculus." ESAIM: Proceedings and Surveys, to appear, doi: $10.1137 / 050641867$.

[11] D.-Q. Lu and H. M. Srivastava, "Some umbral calculus presentations of the Chan-ChyanSrivastava polynomials and the Erkuş-Srivastava polynomials." Comput. Math. Appl., vol. 62, no. 9, pp. 3591-3602, 2011, doi: 10.1137/050641867.

[12] D.-Q. Lu, C.-H. Xiang, and Q.-M. Luo, "Some results for Apostol-type polynomials associated with umbral algebra." Adv. Diff. Equ., vol. 2013, no. 201, 2013, doi: 10.1186/1687-1847-2013201.

[13] Q.-M. Luo and H. M. Srivastava, "Some generalizations of the Apostol-Genocchi polynomials and the Stirling numbers of the second kind." Appl. Math. Comput., vol. 217, no. 12, pp. 5702-5728, 2011, doi: 10.1137/050641867. 
[14] H. Ozden and Y. Simsek, "Unified presentation of $p$-adic $L$-functions associated with unification of the special numbers." preprint, vol. 21, no. 2, pp. 423-441, 2007, doi: 10.1137/050641867.

[15] H. Ozden and Y. Simsek, "Modification and unification of the Apostol-type numbers and polynomials." Appl. Math. Comput., vol. 235, pp. 338-351, 2014, doi: 10.1137/050641867.

[16] H. Ozden, Y. Simsek, and H. M. Srivastava, "A unified presentation of the generating functions of the generalized Bernoulli, Euler and Genocchi polynomials." Comput. Math. Appl., vol. 60, no. 10, pp. 2779-2787, 2010, doi: 10.1137/050641867.

[17] H. Ozden, "Unification of generating function of the Bernoulli, Euler and Genocchi numbers and polynomials." Amer. Inst. Phys. Conf. Proc., vol. 1281, pp. 1125-1128, 2010, doi: 10.1063/1.3497848.

[18] A. Pinter and H. M. Srivastava, "Addition theorems for the Appell polynomials and the associated classes of polynomial expansions." Aequationes Math., vol. 85, pp. 483-495, 2013, doi: $10.1137 / 050641867$.

[19] S. Roman, The Umbral Calculus. New York: Dover Publ. Inc., 2005. doi: 10.1007/978-14614-6946-9.

[20] Y. Simsek, "Generating functions for generalized Stirling type numbers, array type polynomials, Eulerian type polynomials and their applications." arXiv:1111.3848, vol. arXiv:1111.3848, pp. 423-441, 2011, doi: 10.1137/050641867.

[21] Y. Simsek, "Interpolation function of generalized $q$-Bernstein type polynomials and their application, curve and surface." LNCS, vol. 6920, pp. 647-662, 2011, doi: 10.1137/050641867.

[22] Y. Simsek, "Generating functions for generalized Stirling type numbers, array type polynomials, Eulerian type polynomials and their applications." Fixed Point Theory Appl., vol. 2013, no. 87, p. 28 p., 2013, doi: 10.1137/050641867.

[23] H. M. Srivastava, "Some generalizations and basic (or $q$-) extensions of the Bernoulli, Euler and Genocchi polynomials." Appl. Math. Inform. Sci., vol. 5, no. 3, pp. 390-444, 2011, doi: $10.1137 / 050641867$.

[24] H. M. Srivastava and J. Choi, Zeta and q-zeta functions and associated series and integrals. Amsterdam, London, New York: Elsevier Science Publishers, 2012. doi: 10.1007/978-1-4614-69469.

[25] H. M. Srivastava, B. Kurt, and Y. Simsek, "Some families of Genocchi type polynomials and their interpolation functions." Integral Transforms Spec. Funct., vol. 23, no. 12, pp. 919-938, 2012, corrigendum:939-940, doi: 10.1080/10652469.2011.643627.

[26] H. M. Srivastava, K. S. Nisar, and M. A. Khan, "Some umbral calculus presentations of the ChanChyan-Srivastava polynomials and the Erkuş-Srivastava polynomials." Proyecciones J. Math. vol. 33, no. 1, pp. 77-90, 2014, doi: 10.1137/050641867.

\section{Authors' addresses}

Takao Komatsu

Wuhan University, School of Mathematics and Statistics, 430072 Wuhan, China

E-mail address: komat su@whu.edu.cn

\section{Yilmaz Simsek}

University of Akdeniz, Faculty of Science, Department of Mathematics, 07058 Antalya, Turkey

E-mail address: ysimsek@akdeniz.edu.tr 